Staiano, A. E., Beyl, R. A., Hsia, D. S., Katzmarzyk, P. T., \& Newton, R. L. (2018). A 12-week randomized controlled pilot study of dance exergaming in a group: Influence on psychosocial factors in adolescent girls. Cyberpsychology: Journal of Psychosocial Research on Cyberspace, 12(2), article 3. http://dx.doi.org/10.5817/CP2018-2-3

\title{
A 12-week randomized controlled pilot study of dance exergaming in a group: Influence on psychosocial factors in adolescent girls
}

\author{
Amanda E. Staiano, Robbie A. Beyl, Daniel S. Hsia, \\ Peter T. Katzmarzyk, \& Robert L. Newton Jr \\ Pennington Biomedical Research Center, Baton Rouge, Louisiana, USA
}

\begin{abstract}
Dance exergaming, which involves playing an interactive video game that requires the player to make upper and lower body movements by dancing to music, may provide a social physical activity experience that positively impacts psychosocial health. The objective of this randomized controlled study was to examine the effects of groupbased dance exergaming on adolescent girls' psychosocial health including enjoyment, subjective health, perceived peer support, and health-related quality of life. Forty-one adolescents with overweight/obesity were randomly assigned to a 12-week dance exergaming intervention or to a control group. Peer support, subjective health, and health-related quality of life (HRQOL) were assessed pre-and post-intervention, and intervention participants rated enjoyment after each exergaming session. Repeated measures analysis of covariance models controlling for age and baseline body mass index were used to examine condition differences. Results indicated that subjective health improved in the exergaming condition more than control $(p=.02)$. Ratings of peer conflict after the intervention were significantly different by condition $(p=.01)$, with peer conflict stabilizing in the exergaming group and worsening in the control group. There was no difference by condition for HRQOL. Enjoyment remained high throughout the intervention. In summary, group exergaming improved subjective health, stabilized peer conflict, and provided an enjoyable physical activity experience for overweight adolescent girls.
\end{abstract}

Keywords: Quality of life; adolescents; obesity; psychosocial functioning; social skills; development

\section{Introduction}

Adolescents with obesity, who account for $20.5 \%$ of U.S. adolescents (Ogden et al., 2016), engage in less than half of the recommended 60 minutes per day of moderate-to-vigorous physical activity (MVPA; Carson, Staiano, \& Katzmarzyk, 2015). Further, the same study shows disparities by sex and weight status: adolescent girls' physical activity (median of $25.1 \mathrm{~min} /$ day) is about half that of boys' (46.8 min/day), and adolescent girls with obesity engage in half of the physical activity ( $16.9 \mathrm{~min} /$ day) as non-overweight adolescent girls ( $25.5 \mathrm{~min} /$ day) (Carson et al., 2015). Therefore, a focus on physical activity promotion among adolescent girls with obesity is warranted.

Existing research pointed out several barriers that prevent adolescents with obesity from exercising according to the recommendations. Such barriers include lack of peer support to be physically active, perceived incompetence, and perception of the physical activity as not fun (Deforche, De Bourdeaudhuij, \& Tanghe, 2006; Hohepa, Schofield, \& Kolt, 2006). Obesity is associated with many additional challenges, including psychosocial challenges such as difficulty making friends, social isolation, and decreased life satisfaction (Fonseca, Matos, Guerra, \& Pedro, 2009), as well as poor health outcomes including early signs of cardiovascular disease, type 2 diabetes mellitus, nonalcoholic fatty liver disease, and sleep apnea (Kumar \& Kelly, 2017). This constellation of psychosocial and 
physical health challenges contribute to poor health-related quality of life, which is an individual's perceived physical and mental health over time (Ul-Haq, Mackay, Fenwick, \& Pell, 2013).

Physical activity provides a cascade of health benefits especially needed for adolescents with obesity, including chronic disease prevention and improved cardiometabolic health, quality of sleep, improved executive functioning, and reduced risk of depression and anxiety, among others (2018 Physical Activity Guidelines Advisory Committee, 2018). Physical activity may also be a means by which adolescents with obesity can improve their subjective health including their body satisfaction, perceived physical conditioning, and perceived physical selfworth (Goldfield et al., 2007), as well as their health-related quality of life (Staiano et al., 2017a). Identifying enjoyable options for physical activity may therefore be a mechanism to not only promote sustained physical activity but also improve psychosocial health among adolescents with obesity.

\section{Dance Exergaming}

Dance is a physical activity that has a particular appeal to girls and has been used effectively to improve cardiometabolic and psychosocial health among adolescent girls (Robinson et al., 2010). Dancing to music has been shown to be an important predictor of enjoyment within exercise programs (Wininger \& Pargman, 2003), and music contributes to positive mood states during exercise (Lee, 1989). It has been proposed that music can distract from physical discomfort during exercise (Boutcher \& Trenske, 1990).

Newer generation video games that involve dance track the player's movement including their upper and lower body through the use of infra-red sensors and video cameras. These exergames are a popular pastime: nearly one-quarter of 1241 adolescents surveyed reported regularly playing exergames on average 2 days/week for about 50 minutes each bout (O'Loughlin, Dugas, Sabiston, \& O'Loughlin, 2012). Further, in multivariable analyses, exergame players were more likely to be female than male and more likely to be stressed about their weight (O'Loughlin et al., 2012). A second survey among 9125 high school students indicated that 4 of 10 students reported playing exergames and that characteristics associated with playing exergames included having overweight or obesity (Song, Carroll, Lee, \& Fulton, 2015). Players report experiencing fun and enjoyment while playing exergames: in a systematic review of 45 experimental studies, exergames consistently were shown to elicit enjoyment among players (Lee, Kim, Park, \& Peng, 2017). A dance-based exergame was selected for the current study because dance physical activity interventions have demonstrated acceptability and enjoyment by adolescent girls (Robinson et al., 2010).

\section{Dance Exergaming as a Social Activity}

Dance exergames allow players to engage either individually or with partners to model on-screen avatars dancing to popular music. Players earn points for making correct movements to the tempo of the music and can play solitary, competing against themselves for a high score, or can play together with partners to compete against each other. The use of short-term goals within the dance exergame, such as competing against one's own prior scores or against a peer for the highest score in a dance exergame, may provide ongoing opportunities for success that contribute to greater confidence in one's ability to exercise (Yim \& Graham, 2007).

Playing exergames in a group setting where adolescents are dancing together may appeal to youth with obesity who rarely engage in traditional sports due to weight criticism, particularly when the social play is with in-person partners. Social play may promote interactivity and increase player enjoyment, contributing to group cohesion (Vorderer, Hartmann, \& Klimmt, 2003) and subsequently promoting adherence to exercise interventions (Estabrooks, 2000). For instance, a study of twenty-seven 9-12 year-olds found that those assigned to weekly multiplayer dance exergaming classes (a multiplayer interactive dance simulation exergame) played more than twice as long (901 minutes vs. 376 minutes) though the sample size was small and the difference did not reach statistical significance ( $p=.13$; Chin A Paw, Jacobs, Vaessen, Titze, \& van Mechelen, 2008). Further, the youth participating in the multi-player dance exergame group had a significantly lower dropout rate (15\% vs. $64 \%$ ) compared to those who played at home alone (Chin A Paw et al., 2008). Another study demonstrated that youth ages 12 to 18 years playing a tennis exergame engaged more in the exergaming, as measured by higher energy expenditure based on accelerometry, when playing against an in-person partner compared to youth who played the tennis exergame alone (Staiano \& Calvert, 2011). By contrast, a study of 72 children ages 9-12 years examined 
online group play and found no difference in type of play (solitary vs. group) on session duration, frequency, or intensity; exergaming promoted high adherence regardless of whether children played a cycle-based exergame at home alone vs. against other players online (Kaos et al., 2018). However, the Kaos et al. (2018) study utilized online social play whereas the Chin a Paw et al. (2008) and Staiano \& Calvert (2011) used in-person partners. Utilizing in-person social play may elicit more group cohesion and therefore greater engagement in exergaming.

\section{Klub Kinect Social Dance Exergaming Study}

In the "Klub Kinect" pilot and feasibility study, Staiano et al. (2017c) randomly assigned overweight and obese adolescent girls to a 12-week group-based dance exergaming intervention or to a no-treatment control group. Primary outcomes of this intervention indicated that the exergaming did not differentially affect body composition or cardiovascular risk factors in the overall sample compared to the no-treatment control group (Staiano et al., 2017c). On average, adolescents engaged in light intensity physical activity, which is a lower intensity level that involves lower heart rate and energy expenditure compared to MVPA; this engagement in a lower intensity level was likely driven by giving adolescents free choice of intensity and game play among the dance exergames (Staiano, Beyl, Hsia, Katzmarzyk, \& Newton, 2017b). However, among those who engaged in higher intensity physical activity during exergame play, the intervention group significantly reduced abdominal subcutaneous and total adiposity (Staiano et al., 2017c). Further, the intervention participants reported fewer hours of watching TV/videos after the intervention, improved self-efficacy towards physical activity, and scored highly for intrinsic motivation towards exergaming (Staiano et al., 2017b).

The purpose of the present analysis was to examine the effects of this dance exergaming intervention on psychosocial health, as well as to examine quantitative and qualitative feedback regarding the exergaming intervention. It was hypothesized that the social experience of exergaming would improve adolescent girls' subjective health, perceived peer support, and health-related quality of life.

\section{Method}

\section{Participants and Procedure}

Forty-one female adolescents (64\% African American, 36\% White; mean age $16 \pm 1.4$ years) participated in the study. Participants were study volunteers who were recruited via doctors' offices, school nurses, community events, news media, social media sites, and listserv emails. Inclusion criteria included being between 14 and 18 years of age; female; postmenarcheal; body mass index (BMI) indicating overweight or obesity (at or above the $85^{\text {th }}$ percentile; Centers for Disease Control and Prevention, 2016); ability to speak, understand, read, and write English; and willing to accept randomization. Exclusion criteria included being pregnant; hospitalization for mental illness within the past 5 years; impairments that prevented normal ambulation; history or indication of cardiac abnormality, cardiovascular disease, musculoskeletal injuries, or epilepsy; and inability to make the commitment to attend 3 weekly gaming sessions for 12 weeks. Exclusion criteria were determined by a physical examination and medical history conducted by a pediatrician or nurse practitioner. Participants provided written assent, and parents provided written consent. Study procedures were approved by the Pennington Biomedical Research Center Institutional Review Board.

\section{Intervention}

Participants were randomly assigned to a 12-week exergaming intervention or to a no-treatment control condition. Rolling enrollment allowed participants to participate in 12-week windows between January and August 2014. Details on the procedures and assessments were previously published (Staiano et al., 2017b, Staiano et al., 2017c). In brief, intervention participants played exergames together in an exercise classroom within the Pennington Biomedical Research Center's Translation Research Clinic for Children for 60-minute bouts, 3 times/week during the afternoon/early evening using the Just Dance and Dance Central games played on Kinect for Xbox 360. Between 2 and 12 participants exergamed in the room at any given time, with 2 to 3 participants using each exergame console to dance together simultaneously. Further, between 1 and 3 "gaming coaches" provided supervision and ongoing motivation to participants during game play. When there was an odd number of 
participants present, a "gaming coach" played exergames with the participant to ensure participants did not play alone.

Validated instruments were used to assess pre- and post-psychosocial factors at baseline and the final clinic visit. Surveys were administered on a desktop or laptop computer using REDCap electronic data capture tools hosted by the authors' institution. REDCap is a secure, web-based application designed by Vanderbilt University to support data collection and storage for research purposes (Harris et al., 2009). All study participants received $\$ 300$ in compensation throughout the study to offset travel costs and were also offered a 1-h group nutrition counseling session after the final assessment.

\section{Measurements}

Height and weight. Height and weight were measured using a wall-mounted stadiometer and a calibrated scale, respectively. Two measurements were taken for each variable to the nearest 0.1 unit, with a third measurement taken if there was greater than 0.5 unit difference. BMI was calculated as weight in $\mathrm{kg}$ divided by height in meters squared. Baseline BMI was $37.6 \pm 9.3 \mathrm{~kg} / \mathrm{m}^{2}$ in the intervention group and $36.8 \pm 8.9 \mathrm{~kg} / \mathrm{m}^{2}$ in the control group.

Subjective health. Subjective health was measured using a single-item Likert scale ("In general, how would you say your health is: excellent, very good, good, fair, or poor?") (Macias, Gold, Ongur, Cohen, \& Panch, 2015). This single-item self-rating had strong discriminant validity to measure physical health compared to a gold standard general health scale and is sensitive to detect changes in perceived health (Macias et al., 2015). See Table 1 for the pre- and post-intervention values for each measurement by condition.

Table 1. Pre- and post-intervention characteristics of the sample of adolescent girls.

\begin{tabular}{lcccc}
\hline & \multicolumn{2}{c}{ Treatment $(\boldsymbol{n}=\mathbf{2 2})$} & \multicolumn{2}{c}{ Control $(\boldsymbol{n}=\mathbf{1 9})$} \\
\cline { 2 - 5 } & $\begin{array}{c}\text { Pre- } \\
\text { Intervention }\end{array}$ & $\begin{array}{c}\text { Post- } \\
\text { Intervention }\end{array}$ & $\begin{array}{c}\text { Pre- } \\
\text { Intervention }\end{array}$ & $\begin{array}{c}\text { Post- } \\
\text { Intervention }\end{array}$ \\
\hline Subjective Health & $2.6 \pm 0.2$ & $2.9 \pm 0.2$ & $2.7 \pm 0.2$ & $2.6 \pm 0.2$ \\
Peer Support & $73.4 \pm 2.7$ & $72.9 \pm 3.8$ & $70.7 \pm 2.7$ & $73.3 \pm 3.9$ \\
$\quad$ Companionship & $12.7 \pm 0.9$ & $12.6 \pm 1.0$ & $11.4 \pm 0.9$ & $12.2 \pm 1.0$ \\
Conflict & $7.6 \pm 1.0$ & $7.1 \pm 1.0$ & $9.3 \pm 1.0$ & $11.1 \pm 1.0$ \\
Help/aid & $17.5 \pm 0.8$ & $17.1 \pm 1.0$ & $15.8 \pm 3.8$ & $16.4 \pm 1.0$ \\
Security & $13.5 \pm 0.7$ & $14.3 \pm 0.8$ & $13.0 \pm 0.7$ & $14.0 \pm 0.8$ \\
Closeness to Friends & $22.0 \pm 0.8$ & $21.7 \pm 1.3$ & $21.1 \pm 0.8$ & $20.1 \pm 1.3$ \\
Health-Related Quality of Life & $35.7 \pm 1.5$ & $34.2 \pm 1.6$ & $34.2 \pm 1.6$ & $34.4 \pm 1.6$ \\
\hline
\end{tabular}

Note: Values are least squared adjusted means and standard errors from repeated measures analysis of covariance.

Peer support. Peer support was assessed by the 21 -item Friendship Quality Questionnaire (Bukowski, Hoza, \& Boivin, 1994), which includes items that are rated on a 5-point Likert scale where 1 indicates "not true" and 5 indicates "really true." The items are grouped into five domains: companionship, peer conflict, help/aid, security, and closeness to friends, Examples of questions include "My friends think of fun things for us to do together" and "My friends would help me if I needed it." Scores were summed across the 21 -items for a total possible score of 105; sub-scores were also calculated for each domain. Prior data indicated that this survey displayed good internal consistency $(0.71 \leq a \leq 0.86)$ and criterion validity across subscales (Bukowski et al., 1994). See Table 1.

Health-related quality of life. Health-related quality of life was assessed by the KIDSCREEN-10 Index (RavensSieberer et al., 2010). This measure is designed as a single dimension measure to assess quality of life in children and adolescents with chronic illnesses and includes questions about sadness, loneliness, energy, fun with friends, and school performance. The scale includes 10-items with Likert scale from $1=$ "not at all" to 5 = "extremely." Example questions include "Have you felt sad?" and "Have you had fun with your friends?" The scale has shown criterion validity and test-retest reliability (Ravens-Sieberer et al., 2010) and good psychometric properties including in a European cohort of 78,000 youth (Erhart et al., 2009) and an international cohort of 6,106 children (Sampasa-Kanyinga et al., 2017). Per prior published guidelines for scoring (Ravens-Sieberer, 2006), the items were summed and Rasch person parameters were assigned to each sum score, then transformed into values with a mean of 50 and a standard deviation of approximately 10 . See Table 1. 
Enjoyment of exergaming. Enjoyment of exergaming was measured at the end of each exergaming session, with the intervention participant selecting a number between 0 ("Really Did Not Enjoy") and 100 ("Really Enjoyed") in response to the prompt "How I Felt About Playing Today."

Exit survey. An exit survey was administered to and completed by 19 exergaming intervention participants at follow-up. This survey was developed by the research team and included 14 questions. Four questions had closed response options, asking participants if they would continue playing the dance exergames; if yes, how often; if they would play other exergames; if yes, how often. There were 7 items with Likert scale ratings and open text boxes asking "why or why not?" for participants to provide feedback. These items included if they would play dance exergames in school, if they perceived dance exergames as physical activity, if they perceived the dance exergames made them more physically active, if the dance exergames increased their confidence to exercise, if the dance exergames made them have more friends, if the dance exergames made their friendships stronger, and if the dance exergames made them lose weight. The remaining 3 items were open-ended and queried what the participant did and did not like about the dance exergames and if there was any other feedback the participant wished to provide.

\section{Statistical Analysis}

Repeated measures analysis of covariance models controlling for age and baseline BMI were used to examine condition differences in psychosocial factors (i.e. subjective health, components of peer support, and healthrelated quality of life). Paired samples $t$ tests were used to test for changes within each condition. Associations of age, baseline BMI, and BMI change with psychosocial factors were examined using Pearson correlations. Mean enjoyment scores were examined. Exit survey data were summed for the quantitative questions. Qualitative data were examined for common themes and exemplar quotations were selected. Statistical analyses were performed using SAS Version 9.4 (Cary, NC) with two-tailed tests and alpha $=.05$.

\section{Results}

\section{Primary Outcomes}

The participants in the conditions were similar with respect to baseline age ( $M=15.3 \pm 1.2 \mathrm{y}$ in intervention, $M=$ $16.0 \pm 1.4 \mathrm{y}$ in control), BMI, and psychosocial variables (see Table 1). T tests revealed no significant differences between conditions in continuous variables at baseline. Exergaming participants attended on average $77 \pm 24.6 \%$ (range: 28 to 100\%) of the exergaming sessions. Subjective health improved in the exergaming condition more than the control group $\left(p=.02, \eta^{2}=.17\right)$ (see Figure 1). Ratings of peer conflict after the intervention were significantly different by condition $\left(p=.01, \eta^{2}=.05\right)$ : the control condition increased in peer conflict $(p=.03)$ whereas the intervention condition did not (see Figure 2). There were no other condition differences in components of peer support. Age, BMI, and BMI change were not significantly related to any psychosocial factor. There was no significant difference by condition for change in health-related quality of life.

\section{Enjoyment and Motivation}

Self-rated enjoyment averaged $93.3 \pm 3.1$ on a 100 -point scale, and enjoyment remained high throughout the intervention (see Figure 3). All intervention participants (19 of the 19 adolescents) reported they would continue playing exergames after the intervention (16 of 19 reported a few times a week or every day). In response to the question "What did you like about playing Dance Central and Just Dance?" adolescents identified several intrinsic motivators, including "I feel more comfortable dancing now that I have picked up a few moves" (age 15), "Whenever I finish playing I'm in a good mood and I feel good about myself" (age 17), and "Now I push myself to do my exercise daily" (age 16). Social motivators included peer interaction ("I liked playing with other people," age 14) and rapport with the gaming coaches ("[The coaches] made us feel comfortable," age 16; "I felt as if I just left another part of my family," age 15). Nine adolescents reported enjoying the songs and music, and one said she enjoyed exergaming because "it was an alternative towards getting on a tredmill [sic] or the bike" (age 14). One adolescent wrote "I don't want to leave because it's that fun" (age 15). 


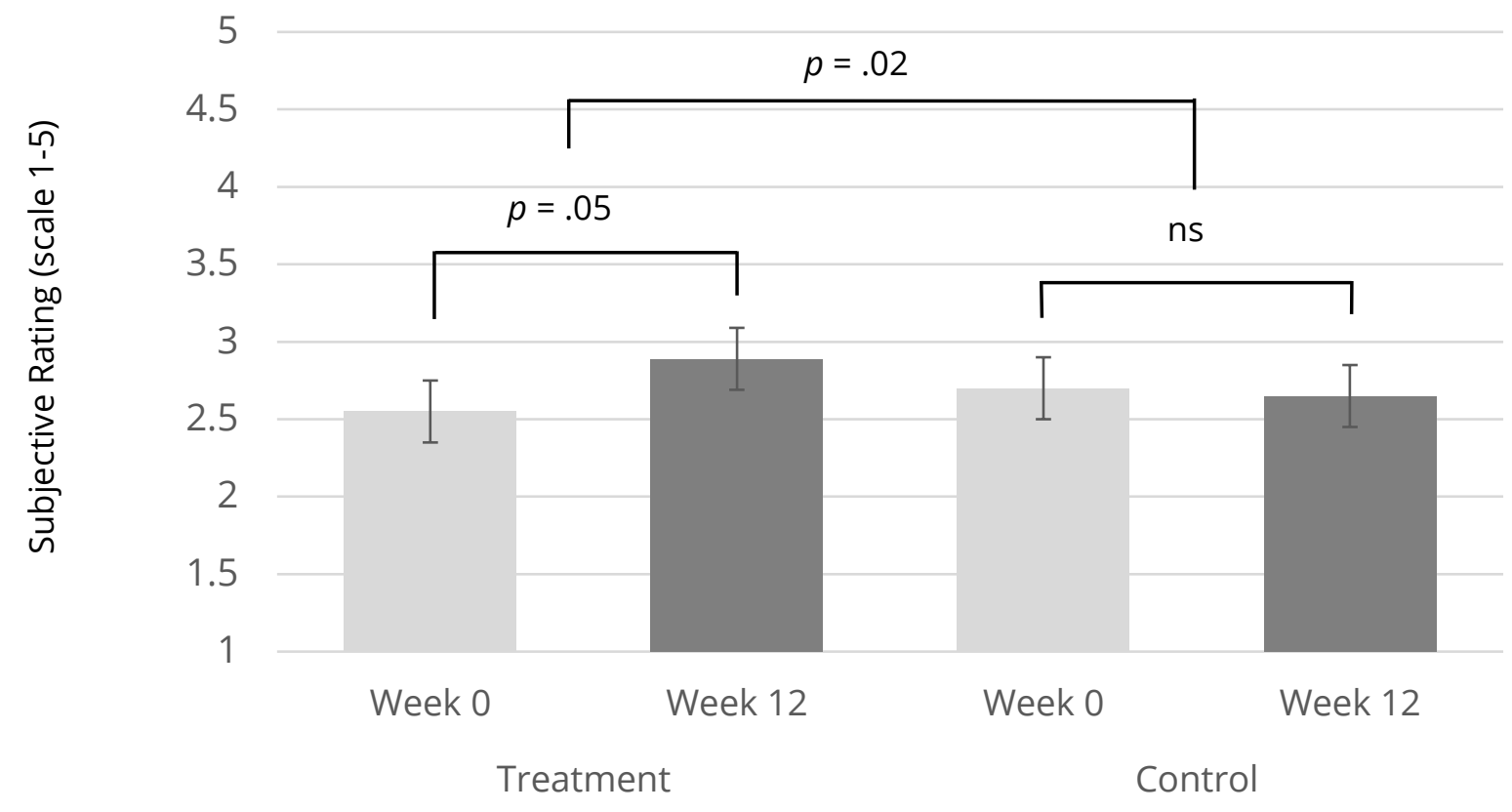

Figure 1. Change in ratings of subjective health following a 12-week group-based exergaming intervention for adolescent girls vs. a no-treatment control.

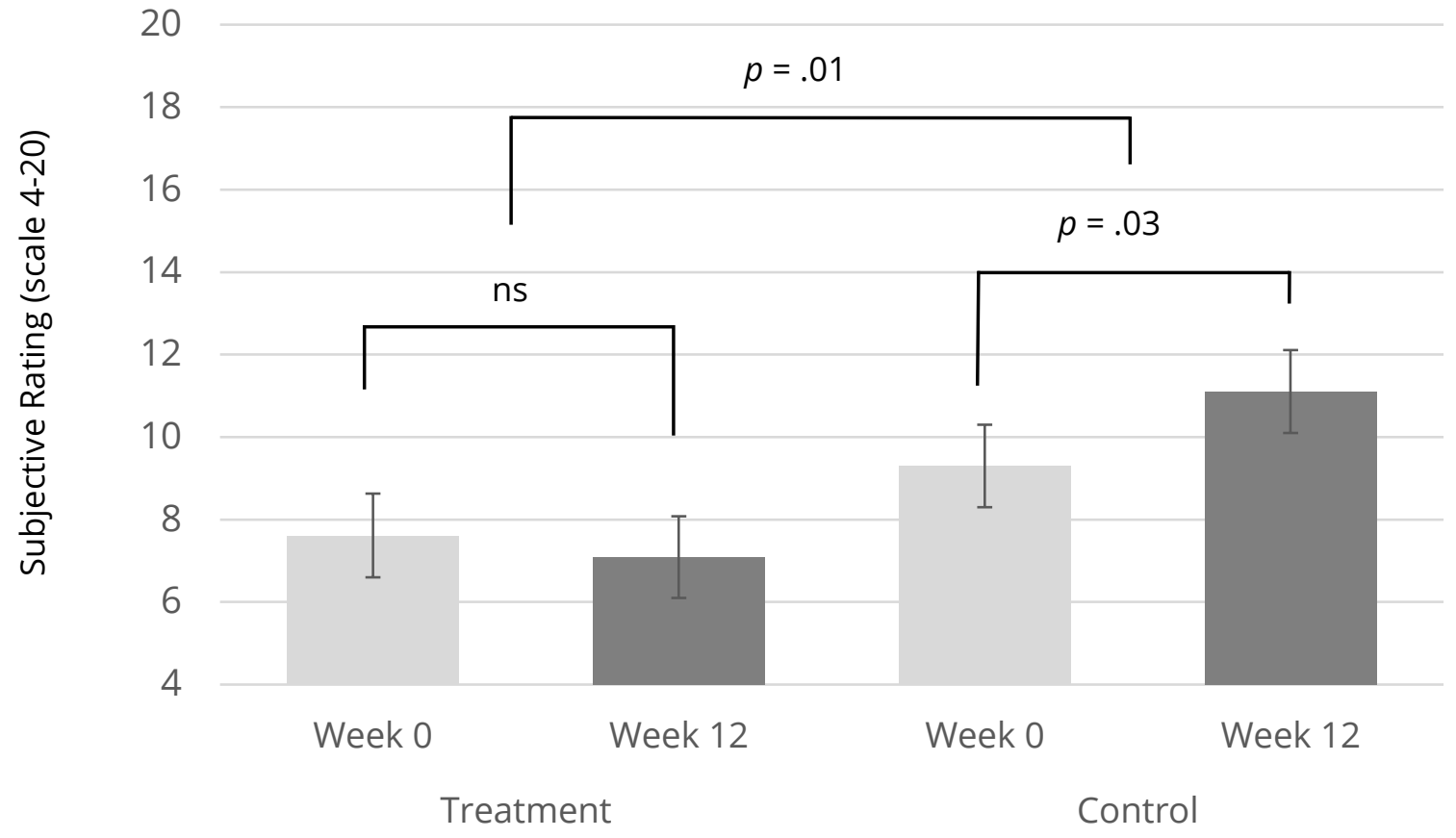

Figure 2. Change in perceptions of peer conflict following a 12-week group-based exergaming intervention for adolescent girls vs. a no-treatment control. 


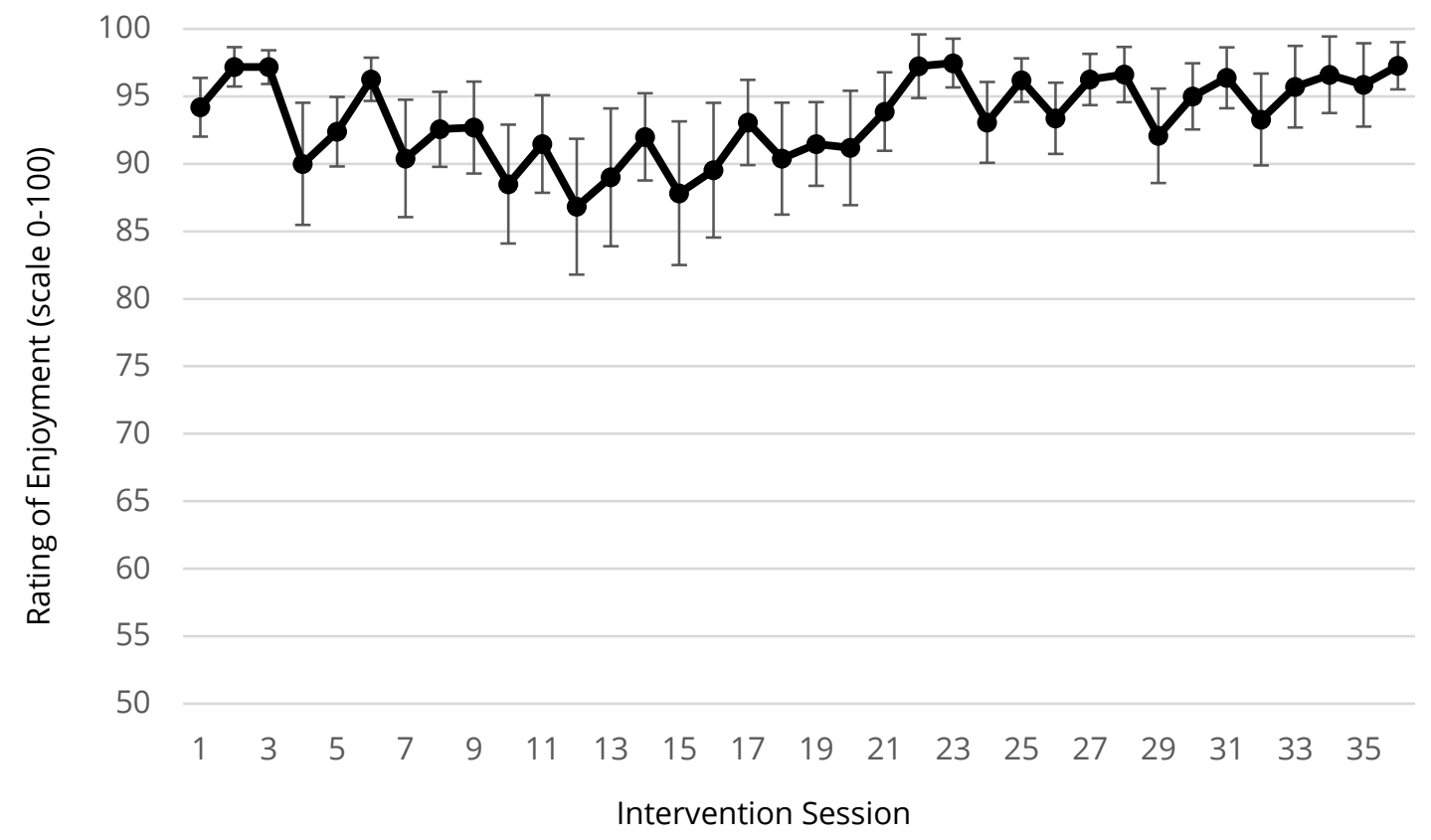

Figure 3. Ratings of enjoyment of across 36 exergaming sessions.

Error bars indicate standard error.

\section{Dislikes about Exergaming}

When asked "What didn't you like about playing Dance Central and Just Dance," the adolescents reported disliking the times and frequency of exergaming required for the intervention and the physical exertion required ("My feet and legs were hurting," age 14). The adolescents reported complaints about the gaming content, including "some of the avatars/dancers looked really creepy and weird" (age 18) and the game "needs more songs" (age 15). There were two complaints about the hardware of the game ("I didn't like that sometimes the camera did not catch me, so I didn't make as many points as I thought I would," age 16).

\section{Sustainment of Exergaming}

The majority (15 of 19, 79\%) reported that they would "really like playing games like Dance Central and Just Dance in physical education classes in school." Reasons provided included enjoying the social interaction of the game play ("it's a fun thing to do with other people," age 15), the novelty of exergaming ("it might attract the attention for most of the people that don't want to do the boring exercise," age 16) and the range of abilities required ("Because it's something everybody can do" [age 14] and "the games ... would allow everyone [sic] age range and weight to be able to dance and enjoy them" [age 16]). One adolescent noted the immersive properties of the game, writing "the time goes by faster and you don't think of it as doing exercise" (age 18). One adolescent who reported she "really wouldn't like playing" the games in school explained "I'd feel like I couldn't dance as much because of people around me" (age 14).

\section{Perception of Exergaming as Physical Activity}

When asked "Do you think playing Dance Central and Just Dance is physical activity?" with response options "it really isn't / sort of isn't / sort of is / really is physical activity," 100\% indicated exergaming "really" or "sort of is" physical activity. Adolescents noted that "you are moving around and dancing, so that is what physical activity is" (age 14) and "it gets you sweating, your heart rate up, etc. all while having fun and dancing" (age 18). One adolescent who said that exergaming "sort of" is physical activity commented "the effort depends on the player" (age 17). Seventeen of 19 (89\%) of adolescents reported exergaming made them more physically active, and 17 of 
19 (89\%) reported exergaming increased exercise confidence. Adolescents responded "I've always danced so it's easier than doing pushups or sit-ups or running a mile" (age 14) and "it made me want to do other physical activities outside of Klub Kinect for instance getting a gym membership" (age 18). Adolescents reported replacing sedentary time with dance ("I don't stay in my room as much as I used to" [age 15] and "I used to spend that hour either laying down watching tv or just simply not doing anything" [age 17]). In regards to confidence, adolescents reported "I feel more comfortable dancing now that I have picked up a few moves" (age 15) and "[exergaming] helped me know that it doesn't matter what size you are you can still do it" (age 16).

\section{Social Relationships}

The majority of participants (13 of 19,68\%) reported playing the dance exergames "really" or "sort of" made them have more friends. Reasons included "we'd have fun competing with one another" (age 15), "we liked the same songs and dances" (age 14), and "it made it easier to meet new people" (age 17). For those who did not think exergaming made them have more friends, reasons included "I'm not a very sociable person" (age 17) and "I knew no one and the experience was totally new to me" (age 16).

\section{Discussion}

Exergames have been proposed as a method to motivate physically inactive people to engage in physical activity (Yim \& Graham, 2007). The Klub Kinect study involved 36 hours of light intensity physical activity over a 12-week period (Staiano et al., 2017b) and produced high levels of enjoyment by the adolescent players throughout the intervention. The adolescent girls perceived that exergaming was physical activity and reported that exergaming made them more physically active.

The findings reported herein are among the first to assess the impact of group dance exergaming on adolescent girls' psychosocial health, including peer support, subjective health, and quality of life. Whereas prior publications focused on the intervention's effects on physical activity, screen-time, self-efficacy, and intrinsic motivation (Staiano et al., 2017b; Staiano et al., 2017c), the present study examined the behavioral mechanisms of these effects via the role of enhanced social interaction and peer support. Compared to the control group, the dance exergaming intervention stabilized perceptions of peer conflict and improved ratings of subjective health. Based on enjoyment ratings throughout the intervention coupled with exit surveys at the end of the intervention, adolescents reported that exergaming provided an enjoyable way to exercise together with peers in a nonthreatening environment. Feelings of social cohesion are associated with higher physical activity levels among adolescents (Utley, Affuso, \& Rucks, 2016). Many of the adolescents in the exergaming condition reported enjoying the fun of competing with peers, and the majority reported exergaming made them have more friends. These findings extend evidence from a prior trial that involved adolescent boys and girls exergaming approximately 1 hour/week over a 20-week period which elicited intrinsic motivation (Staiano, Abraham, \& Calvert, 2013) and increased peer support (Staiano et al., 2013). The present study observed reduced peer conflict in the exergaming vs. control group in a shorter time period but with higher frequency of exergaming.

There was no observed effect of this dance exergaming intervention on health-related quality of life compared to the control group. Health-related quality of life encompasses both physical and social functioning, both of which are lower among adolescents with obesity compared to normal weight peers (Keating, Moodie, \& Swinburn, 2011). While the 10-item version of the KIDSCREEN Index has shown criterion validity and test-retest reliability (RavensSieberer et al., 2010), with 10 items there is less variability than longer scales, which may have resulted in null effects. Other instruments such as the PedsQL are more commonly used and may provide more in-depth ratings of domains that are sensitive to changes and of importance to adolescents with obesity such as physical, emotional, and social functioning (Buttitta, Iliescu, Rousseau, \& Guerrien, 2014). While ratings of quality of life did not change, the adolescents who exergamed improved their subjective health rating, which has been identified as an important outcome measure in obesity interventions (National Institutes of Health, 2011) and a simple method to track self-perceptions of health and well-being (Macias et al., 2015). Peer conflict stabilized in the exergaming group compared to the control group, but there were no detectable benefits in social functioning aside from qualitative responses. Furthermore, as previously published, the intervention did not improve cardiovascular risk factors and only reduced adiposity among those who engaged in higher physical activity (Staiano et al., 2017c). Therefore, the physical health improvements may have been insufficient to change perceptions of quality of life. 
Nevertheless, quality of life was identified as a fundamental measure of population health in the Healthy People 2020 Report (U.S. Department of Health and Human Services, 2016), and future research should identify ways to improve health-related quality of life among the adolescent population (Kolodziejczyk et al., 2015).

Enjoyment was consistently high across the 12-week intervention within the exergaming group. Other trials observed that adolescents and young adults report higher enjoyment of exergaming vs. traditional physical activity (Duncan \& Dick, 2012; Garn, Baker, Beasley, \& Solmon, 2012; Graves et al., 2010). Future research should determine if exergaming elicits additional motivation for sustained physical activity among adolescents with obesity in conjunction with traditional physical activity equipment, particularly among those youth who enjoy exergaming. However, because of the short-term (12-week) nature of the study with no follow-up assessment, it is not possible to examine sustainability of dance exergaming after the end of the program. The lack of follow-up assessment is an important limitation of current physical activity studies.

Researchers have identified several ways to decrease barriers to physical activity among adolescents with obesity, including the use of separate exercise sessions for adolescents with obesity, finding ways to make the exercise experience more fun, promoting the improvement of self-efficacy towards exercise, and emphasizing the wide range of health benefits of exercise beyond weight loss (Daley, Copeland, Wright, \& Wales, 2008). The Klub Kinect intervention was designed to provide each of these elements: the inclusion of only adolescent girls with overweight or obesity which may have improved the participants' comfort while exercising, its use of dance exergaming that was highly enjoyed by the adolescent girls, its improvement of the adolescents' self-efficacy towards exercise (Staiano et al., 2017b), and reports by the adolescents that they enjoyed a more positive mood and social connections to coaches and peers during the dance exergaming. While exergaming has also shown promise to increase physical activity in a home setting (Staiano et al., 2018), the unique aspect of the present study is that it provided an exercise classroom setting to gather adolescent girls, who were all overweight or had obesity, from throughout the community to play together.

Participants noted several motivators from the dance exergaming intervention that have been previously identified as motivators for exercise programs (Yim \& Graham, 2007). First, upbeat music was integrated into exergame play, which has been shown to be an important predictor of enjoyment within exercise programs (Wininger \& Pargman, 2003) and contributes to positive mood states during exercise (Lee, 1989). Several participants named the music and songs as a main reason for enjoying the exergaming, though one noted a limited song selection as a complaint.

A second motivator was the use of "gaming coaches" as instructors to facilitate group rapport and motivate continued exergaming. Instructors who provide ongoing support and encouragement are an important factor predicting the enjoyment of aerobics classes (Wininger \& Pargman, 2003). Importantly, in the Klub Kinect study the coaches were an adjunct to the exergames, indicating that having an adult such as a physical education teacher is important to motivate and supervise exergame play. Prior research has indicated that introducing dance exergames in a physical education course increased students' use of exergames outside of class, thereby indicating the influence of modeling behaviors to change adolescents' overall physical activity (Quinn, 2013). The role of a gaming coach could be filled by a physical education teacher, parent, or lay health coach; therefore, there is potential for broader dissemination by providing exergaming consoles to groups of adolescents in schools, recreational facilities, or other public spaces.

A third motivating aspect was that the intervention participants selected the intensity and challenge of the songs and thereby could start at a beginner's level and increase in difficulty. Allowing a sense of control over intensity of exergaming and mastery of gaming content has been noted as a motivational aspect of exergames (Roemmich, Lambiase, McCarthy, Feda, \& Kozlowski, 2012; Wagener, Fedele, Mignogna, Hester, \& Gillaspy, 2012). Furthermore, greater feelings of control over gameplay has been related to higher enjoyment (Limperos, Schmierbach, Kegerise, \& Dardis, 2011). Adolescents were regularly provided with positive reinforcement by the dance exergames (e.g. "Flawless" and "Almost" flashed on the screen), and success occurred at regular intervals after each individual song. Frequent intermittent reinforcement may act as a mechanism that makes the exergaming enjoyable and sustainable for longer-term health benefits. 
Finally, the extent to which social play does or does not facilitate motivation as a means to promote sustained physical activity warrants further exploration. Cooperative, social exergame play has been shown to be more effective for weight loss in overweight and obese adolescents compared to competitive play (Staiano et al., 2013). By contrast, parallel competition in separate physical spaces resulted in higher enjoyment and motivation for future play compared to cooperating with another player in the same physical space or playing solitary (Peng \& Crouse, 2013), likely due to space limitations. In the present study, when provided a choice of dance exergames to play within a social, group-based setting, the adolescents selected light intensity physical activity rather than MVPA (Staiano et al., 2017b). Future research should examine how to elicit higher levels of difficulty and intensity within exergame play when sustained physical activity engagement is the goal. Further, there is a dearth of literature on sustained effects of technology-facilitated physical activity interventions on adolescents' behaviors; a prior publication of data from this study observed no changes in accelerometry-measured physical activity outside of the intervention (Staiano et al., 2017b). Removing access to the exergames returned adolescents' physical activity levels to baseline, which calls for future intervention research to examine ways to sustain effects beyond the intervention.

Limitations of this study include a small sample size, no long-term follow-up to test for sustained effects, and limited external validity due to the supervised laboratory setting. The age range of 14 to 18 years is a wide developmental period and may impede social connections due to differing education and maturation levels. Participants were study volunteers who selected into the dance exergaming trial; therefore, selection bias is likely. Because a "no treatment" control group was selected as the comparator as opposed to an attention-matched control, it is not possible to distinguish observed effects from the attention provided by the research staff who acted as gaming coaches or the effects of being enrolled in an intervention regardless of the content of the intervention. Clinical psychological symptoms and other social support beyond peer support were not measured in the present study. A 10-week dance-based exergaming trial in 40 obese adolescents indicated an improvement in parental relations coupled with reduced internalizing and externalizing symptomatology (Wagener et al., 2012). Parents were not involved in the Klub Kinect study other than providing transportation (except for adolescents who drove themselves); therefore, parental involvement is an area of future research to further improve adolescents' social relations beyond peers.

\section{Conclusions}

Creating a social environment for physical activity through dance exergames improved subjective health, stabilized peer conflict, and was an enjoyable activity. Social exergaming may be a method by which technology creates a positive environment for adolescent girls to engage in sustained physical activity.

\section{Acknowledgement}

This work was supported by cores and resources funded by a National Institute of Diabetes and Digestive and Kidney Diseases of the National Institutes of Health Center Grant entitled "Nutritional Programming: Environmental and Molecular Interactions" (\# P30DK072476). AES and PTK are supported, in part, by the Louisiana Clinical \& Translational Sciences Center (LA CaTS) of the National Institute of General Medical Science of the National Institutes of Health (NIGMS \#1U54GM104940). PTK is supported, in part, by the Marie Edana Corcoran Endowed Chair in Pediatric Obesity and Diabetes. The funding sources had no involvement in study design; collection, analysis, or interpretation of data; writing the report; or decision to submit the article for publication. 


\section{References}

2018 Physical Activity Guidelines Advisory Committee. (2018). 2018 physical activity guidelines advisory committee scientific report. Retrieved from https://health.gov/paguidelines/second-

edition/report/pdf/PAG_Advisory_Committee_Report.pdf

Boutcher, S. H., \& Trenske, M. (1990). The effects of sensory deprivation and music on perceived exertion and affect during exercise. Journal of Sport and Exercise Psychology, 12, 167-176.

https://dx.doi.org/10.1123/jsep.12.2.167

Bukowski, W. M., Hoza, B., \& Boivin, M. (1994). Measuring friendship quality during pre-and early adolescence: The development and psychometric properties of the Friendship Qualities Scale. Journal of Social and Personal Relationships, 11, 471-484. https://doi.org/10.1177/0265407594113011

Buttitta, M., Iliescu, C., Rousseau, A., \& Guerrien, A. (2014). Quality of life in overweight and obese children and adolescents: A literature review. Quality of Life Research, 23, 1117-1139. https://doi.org/10.1007/s11136-013-05685

Carson V., Staiano A. E., \& Katzmarzyk P. T. (2015). Physical activity, screen time, and sitting among U.S. adolescents. Pediatric Exercise Science, 27, 151-159. https://doi.org/10.1123/pes.2014-0022

Centers for Disease Control and Prevention. (2016). A SAS program for the 2000 CDC growth charts (ages 0 to $<20$ years). Retrieved from www.cdc.gov/nccdphp/dnpao/growthcharts/resources/sas.htm

Chin A Paw, M. J. M., Jacobs, W. M., Vaessen, E., Titze, S., \& van Mechelen, W. (2008) The motivation of children to play an active video game. Journal of Science and Medicine in Sport, 11, 163-166.

https://doi.org/10.1016/j.jsams.2007.06.001

Daley, A. J., Copeland, R., Wright, N., \& Wales, J. K. (2008). I can actually exercise if I want to; it isn't as hard as I thought: A qualitative study of the experiences and views of obese adolescents participating in an exercise therapy intervention. Journal of Healthy Psychology, 13, 810-819. https://doi.org/10.1177/1359105308093865

Deforche, B. I., De Bourdeaudhuij, I. M., \& Tanghe, A. P. (2006) Attitude toward physical activity in normal-weight, overweight and obese adolescents. Journal of Adolescent Health, 38, 560-568.

https://doi.org/10.1016/j.jadohealth.2005.01.015

Duncan, M. J., \& Dick, S. (2012). Energy expenditure and enjoyment of exergaming: A comparison of the Nintendo Wii and the gamercize power stepper in young adults. Journal of Adolescent Health 16, 92-98. https://doi.org/10.5604/17342260.1011386

Erhart, M., Ottova, V., Gaspar, T., Jericek, H., Schnohr, C., Alikasifoglu, M., ... Ravens-Sieberer, U. (2009). Measuring mental health and well-being of school-children in 15 European countries using the KIDSCREEN-10 Index. International Journal of Public Health, 54, 160-166. https://doi.org/10.5604/17342260.1011386

Estabrooks, P. A. (2000). Sustaining exercise participation through group cohesion. Exercise and Sport Sciences Reviews, 28, 63-67.

Fonseca, H., Matos, M. G., Guerra, A., \& Pedro, J. G. (2009). Are overweight and obese adolescents different from their peers? International Journal of Pediatric Obesity, 4, 166-174. https://doi.org/10.1080/17477160802464495

Garn, A. C., Baker, B. L., Beasley, E. K., \& Solmon, M. A. (2012). What are the benefits of a commercial exergaming platform for college students? Examining physical activity, enjoyment, and future intentions. Journal of Physical Activity \& Health, 9, 311-318. https://doi.org/10.1123/jpah.9.2.311 
Goldfield, G. S., Mallory, R., Parker, T., Cunningham, T., Legg, C., Lumb, A., .. Adamo, K. B. (2007). Effects of modifying physical activity and sedentary behavior on psychosocial adjustment in overweight/obese children. Journal of Pediatric Psychology, 32, 783-793. https://doi.org/10.1093/jpepsy/jsm017

Graves, L. E., Ridgers, N. D., Williams, K., Stratton, G., Atkinson, G. T., \& Cable, N. T. (2010). The physiological cost and enjoyment of Wii Fit in adolescents, young adults, and older adults. Journal of Physical Activity and Health, 7, 393-401. https://doi.org/10.1123/jpah.7.3.393

Harris, P. A., Payne, M., Taylor, R., Conde-Glez, C., Conde, G., Gonzalez, N., \& Thielke, R. (2009). Research electronic data capture (REDCap)-A metadata-driven methodology and workflow process for providing translational research informatics support. Journal of Biomedical Informatics, 42, 377-381.

https://doi.org/10.1016/j.jbi.2008.08.010

Hohepa, M., Schofield, G., \& Kolt, G. S. (2006). Physical activity: What do high school students think? Journal of Adolescent Health, 39, 328-336. https://doi.org/10.1016/j.jadohealth.2005.12.024

Kaos, M. D., Beauchamp, M. R., Bursick, S., Latimer-Cheung, A. E., Hernandez, H., Warburton, D. E. R., ... Rhodes, R. E. (2018). Efficacy of online multi-player versus single-player exergames on adherence behaviors among children: A nonrandomized control trial. Annals of Behavioral Medicine, 52, 878-889.

https://doi.org/10.1093/abm/kax061

Keating, C. L., Moodie, M. L., \& Swinburn, B. A. (2011). The health-related quality of life of overweight and obese adolescents - a study measuring body mass index and adolescent-reported perceptions. International Journal of Pediatric Obesity, 6, 434-441. https://doi.org/10.3109/17477166.2011.590197

Kolodziejczyk, J. K., Gutzmer, K., Wright, S. M., Arredondo, E. M., Hill, L., Patrick, K., . . Norman, G. J. (2015). Influence of specific individual and environmental variables on the relationship between body mass index and health-related quality of life in overweight and obese adolescents. Quality of Life Research, 24, 251-261. https://doi.org/10.1007/s11136-014-0745-1

Kumar, S. \& Kelly, A. (2017). Review of childhood obesity: From epidemiology, etiology, and comorbidities to clinical assessment and treatment. Mayo Clinic Proceedings, 92, 251-265.

https://doi.org/10.1016/j.mayocp.2016.09.017

Lee, K. P. (1989). The effects of musical tempos on psychophysical responding during sub-maximal treadmill running (Unpublished master's thesis). Pennsylvania State University, Pennsylvania, US.

Lee, S., Kim, W., Park, T., \& Peng, W. (2017). The psychological effects of playing exergames: A systematic review. Cyberpsychology, Behavior, and Social Networking, 20, 513-532. https://doi.org/10.1089/cyber.2017.0183

Limperos, A. M., Schmierbach, M. G., Kegerise, A. D., \& Dardis, F. E. (2011). Gaming across different consoles: Exploring the influence of control scheme on game-player enjoyment. Cyberpsychology, Behavior, and Social Networking, 14, 345-350. https://doi.org/10.1089/cyber.2010.0146

Macias, C., Gold, P. B., Ongur, D., Cohen, B. M., \& Panch, T. (2015). Are single-item global ratings useful for assessing health status? Journal of Clinical Psychology in Medical Settings, 22, 251-264.

https://doi.org/10.1007/s10880-015-9436-5

National Institutes of Health. (2011). Strategic Plan for NIH Obesity Research. Retrieved from https://www.obesityresearch.nih.gov/about/strategic-plan.aspx

Ogden, C. L., Carroll, M. D., Lawman, H. G., Fryar, C. D., Kruszon-Moran, D., Kit, B. K., \& Flegal, K. M. (2016). Trends in obesity prevalence among children and adolescents in the United States, 1988-1994 through 2013-2014. JAMA, 315, 2292-2299. https://doi.org/10.1001/jama.2016.6361 
O'Loughlin, E. K., Dugas, E. N., Sabiston, C. M., \& O'Loughlin, J. L. (2012). Prevalence and correlates of exergaming in youth. Pediatrics, 130, 806-814. https://doi.org/10.1542/peds.2012-0391

Peng, W., \& Crouse, J. (2013). Playing in parallel: The effects of multiplayer modes in active video game on motivation and physical exertion. Cyberpsychology, Behavior, and Social Networking, 16, 423-427.

https://doi.org/10.1089/cyber.2012.0384

Quinn, M. (2013). Introduction of active video gaming into the middle school curriculum as a school-based childhood obesity intervention. Journal of Pediatric Health Care, 27, 3-12.

https://doi.org/10.1016/j.pedhc.2011.03.011

Ravens-Sieberer, U., Erhart, M., Rajmil, L., Herdman, M., Auquier, P., Bruil, J., . . . Kilroe, J. (2010). Reliability, construct and criterion validity of the KIDSCREEN-10 score: A short measure for children and adolescents' wellbeing and health-related quality of life. Quality of Life Research, 19, 1487-1500. https://doi.org/10.1007/s11136010-9706-5

Ravens-Sieberer, U. (2006). The KIDSCREEN questionnaires: Quality of life questionnaires for children and adolescents; Handbook. Lengerich: Pabst Science Publisher.

Robinson, T. N., Matheson, D. M., Kraemer, H. C., Wilson, D. M, Obarzanek, E., Thompson, N. S., ... Killen, J. D. (2010). A randomized controlled trial of culturally tailored dance and reducing screen time to prevent weight gain in low-income African American girls: Stanford GEMS. Archives of Pediatrics and Adolescent Medicine, 164, 9951004. https://doi.org/10.1001/archpediatrics.2010.197

Roemmich, J. N., Lambiase, M. J., McCarthy, T. F., Feda, D. M., \& Kozlowski, K. F. (2012). Autonomy supportive environments and mastery as basic factors to motivate physical activity in children: a controlled laboratory study. International Journal of Behavioral Nutrition and Physical Activity, 9, article 16. https://doi.org/10.1186/14795868-9-16

Sampasa-Kanyinga, H., Standage, M., Tremblay, M. S., Katzmarzyk, P. T., Hu, G., Kuriyan, R., ... Chaput, J. P. (2017). Associations between meeting combinations of 24-h movement guidelines and health-related quality of life in children from 12 countries. Public Health, 153, 16-24. https://doi.org/10.1016/j.puhe.2017.07.010

Song, M., Carroll, D. D., Lee, S. M., \& Fulton, J. E. (2015). Active gaming among high school students-United States, 2010. Games for Health Journal, 4, 325-331. https://doi.org/10.1089/g4h.2014.0126

Staiano, A. E., Abraham, A. A., \& Calvert, S. L. (2013). Adolescent exergame play for weight loss and psychosocial improvement: A controlled physical activity intervention. Obesity, 21, 598-601. https://doi.org/10.1002/oby.20282

Staiano, A. E., Beyl, R. A., Hsia, D. S., Jarrell, A. R., Katzmarzyk, P. T., Mantzor, S., . . Tyson, P. (2017a). Step tracking with goals increases children's weight loss in behavioral intervention. Childhood Obesity, 13, 283-290.

https://doi.org/10.1089/chi.2017.0047

Staiano, A. E., Beyl, R. A., Hsia, D. S., Katzmarzyk, P. T., \& Newton Jr., R. L. (2017b). Twelve weeks of dance exergaming in overweight and obese adolescent girls: Transfer effects on physical activity, screen-time, and selfefficacy. Journal of Sport and Health Science, 6, 4-10. https://doi.org/10.1016/j.jshs.2016.11.005

Staiano, A. E., \& Calvert, S. L. (2011). Wii tennis play for low-income African American adolescents' energy expenditure. Cyberpsychology: Journal of Psychosocial Research on Cyberspace, 5(1). Retrieved from: https://cyberpsychology.eu/article/view/4241/3287

Staiano, A. E., Marker, A. M., Beyl, R. A., Hsia, D. S., Katzmarzyk, P. T., \& Newton Jr., R. L. (2017c). A randomized controlled trial of dance exergaming for exercise training in overweight and obese adolescent girls. Pediatric Obesity, 12, 120-128. https://doi.org/10.1111/ijpo.12117 
Staiano, A. E., Beyl, R. A., Guan, W., Hendrick, C. A., Hsia, D. S., \& Newton Jr., R. L. (2018). Home-based exergaming among children with overweight and obesity: A randomized controlled trial. Pediatric Obesity, 13, 724-733.

https://doi.org/10.1111/ijpo.12438

Ul-Haq, Z. Mackay, D. F., Fenwick, E., \& Pell, J. P. (2013). Meta-analysis of the association between body mass index and health-related quality of life among children and adolescents, assessed using the pediatric quality of life inventory index. The Journal of Pediatrics, 162, 280-286. https://doi.org/10.1016/j.jpeds.2012.07.049

U.S. Department of Health and Human Services. Healthy People 2020 - Improving the Health of Americans. Retrieved from: http://healthypeople.gov/2020

Utley, J. M., Affuso, O., \& Rucks, A. C. (2016). Adolescent obesity in contextual settings: A scoping study of multilevel and hierarchical examinations. Clinical Obesity, 6, 296-304. https://doi.org/10.1111/cob.12163

Vorderer, P., Hartmann, T., \& Klimmt, C. (2003). Explaining the enjoyment of playing video games: The role of competition. In ICEC '03 Proceedings of the second international conference on Entertainment computing (pp. 1-9). Pittsburgh, PA: Carnegie Mellon University.

Wagener, T. L., Fedele, D. A., Mignogna, M. R., Hester, C. N., \& Gillaspy, S. R. (2012). Psychological effects of dance-based group exergaming in obese adolescents. Pediatric Obesity, 7, e68-e74.

https://doi.org/10.1111/j.2047-6310.2012.00065.x

Wininger, S. R., \& Pargman, D. (2003). Assessment of factors associated with exercise enjoyment. Journal of Music Therapy, 40, 57-73. https://doi.org/10.1093/jmt/40.1.57

Yim, J., \& Graham, T. (2007) Using games to increase exercise motivation. In Proceedings of the 2007 conference on Future Play 2007 (pp. 166-173). Toronto, ON, Canada. https://doi.org/10.1145/1328202.1328232

\section{Correspondence to:}

Amanda E. Staiano

Pennington Biomedical Research Center

6400 Perkins Road

Baton Rouge 70808

LA, USA

Email: amanda.staiano(at)pbrc.edu

Editorial record: First submission received on January 25, 2018. Revisions received on October 17, 2018, and November 16, 2018. Accepted for publication on November 24, 2018.

The article is part of the Special Issue "Health and Technology" guest edited by Michal Molcho (NUI Galway), Christine Pellegrini (University of South Carolina), and Jana Holubcikova (PJ Safarik University). The manuscript was originally submitted to the journal's regular issue. 


\section{About Authors}

Amanda E. Staiano, PhD is Director of the Pediatric Obesity and Health Behavior Laboratory at Pennington Biomedical Research Center. Dr. Staiano is a developmental psychologist in pediatric obesity with an interest in technology-mediated physical activity interventions and the integration of these technologies into the behavioral treatment of childhood obesity. Her research has examined how technological devices like exergames (i.e. activitypromoting video games) affect youths' adiposity, physical activity, and eating behaviors.

Robbie A. Beyl, PhD is Assistant Professor in Biostatistics at Pennington Biomedical Research Center. Dr. Beyl's interests are in statistical consulting and educating the research community on the importance of statistics, particularly post-doctoral researchers and junior faculty. Statistical research interests include the development of statistical methods for the analysis of categorical data and the application of novel statistical techniques to the design, analysis and interpretation of data.

Daniel S. Hsia, MD is Assistant Professor and a dual-certified pediatric and adult endocrinologist at Pennington Biomedical Research Center. Dr. Hsia's research focuses on prevention and intervention strategies for diabetes and obesity across the lifespan. He is especially interested in expanding treatment options for children with diabetes and obesity by translating approved therapies into the pediatric population.

Peter T. Katzmarzyk, PhD is Professor, Marie Edana Corcoran Endowed Chair in Pediatric Obesity and Diabetes, Associate Executive Director for Population and Public Health Sciences, and directs the Physical Activity and Obesity Epidemiology Laboratory at Pennington Biomedical Research Center. Dr. Katzmarzyk is an internationally recognized leader in the field of physical activity and obesity, with a special emphasis on pediatrics and ethnic health disparities. He has over two decades of experience in conducting large clinical and population-based studies in children and adults. In addition to his research, Dr. Katzmarzyk plays a leading role in national health advocacy initiatives. He chairs the Research Advisory Committee for the U.S. Report Card on Physical Activity for Children and Youth for the National Physical Activity Plan Alliance. He also serves on the 2018 U.S. Physical Activity Guidelines Advisory Committee for the U.S. Department of Hea Ith and Human Services.

Robert L. Newton Jr, PhD is Associate Professor and Director of the Physical Activity and Ethnic Minority Health Laboratory at Pennington Biomedical Research Center. Dr. Newton's research interests center on addressing health disparities in African American children, adults, and older adults. Although much of his work involves developing physical activity promotion interventions, he also engages in weight loss studies. Dr. Newton utilizes community-based and technology-based interventions to address health disparities. 